\title{
Performances of the gas pixel detector to a continuum and highly polarized x-ray beam
}

\begin{abstract}
Alessia Tortosa, Sergio Fabiani, Paolo Soffitta, Fabio Muleri, Mario Cirillo, et al.
\end{abstract}

Alessia Tortosa, Sergio Fabiani, Paolo Soffitta, Fabio Muleri, Mario Cirillo, Riccardo Ferrazzoli, Alda Rubini, Antonio Tobia, Alessandro Trinca, "Performances of the gas pixel detector to a continuum and highly polarized x-ray beam," Proc. SPIE 10699, Space Telescopes and Instrumentation 2018: Ultraviolet to Gamma Ray, 106995A (6 July 2018); doi: 10.1117/12.2313741

SPIE Event: SPIE Astronomical Telescopes + Instrumentation, 2018, Austin, Texas, United States 


\title{
Performance of the gas pixel detector to a continuum and highly polarized X-ray beam
}

\author{
Alessia Tortosa ${ }^{a}$, Sergio Fabiani ${ }^{a}$, Paolo Soffitta ${ }^{a}$, Fabio Muleri $^{a}$, Mario Cirillo $^{a}$, Riccardo \\ Ferrazzoli $^{a}$, Alda Rubini ${ }^{a}$, Antonino Tobia ${ }^{a}$ and Alessandro Trinca ${ }^{a}$ on the behalf of the IXPE \\ Team. \\ ${ }^{a}$ INAF-IAPS, via del fosso del Cavaliere 100, I-00133, Rome, Italy.
}

\begin{abstract}
We report on the first measure of the polarization of a laboratory source with a continuum energy spectrum, which simulates the effect of a real astrophysical source, carried out with a prototype of the Gas Pixel Detector (GPD). This detector is an X-ray polarimeter exploiting the photoelectric effect both to measure the polarization and to obtain the image of astrophysical sources. The gas pixel detector will be the focal plane detector on board the IXPE (Imaging X-ray Polarimetry Explorer) mission selected by NASA in the framework of the Explorer program for a launch in 2021.
\end{abstract}

Keywords: IXPE, GPD, X-ray polarimetry, imaging

\section{INTRODUCTION}

The Gas Pixel Detector (GPD), ${ }^{12}$ is an X-ray polarimeter that exploits the photoelectric effect to measure the polarization and to obtain the image of astrophysical sources (see Fig.1 for a schematic view). By means of the analysis of the statistical moments of photoelectron tracks in gas it derives the direction of emission of the photoelectrons that is more probable along the photon polarization direction (following a $\cos (\theta)^{2}$ modulation).

$\mathrm{X}$-ray photons are absorbed in a $1 \mathrm{~cm}$ thick gas cell filled with a He-DME gas mixture and the absorption

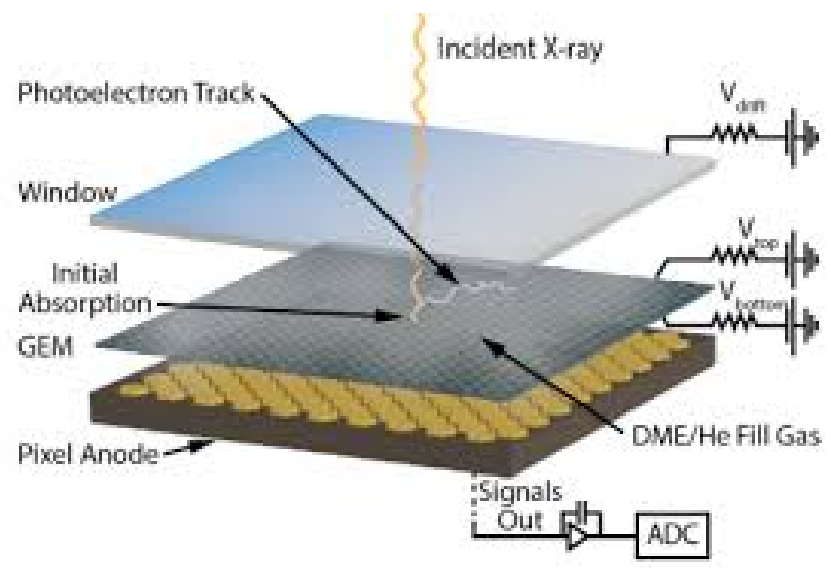

Figure 1. Schematic view of the GPD

points are calculated by means of the analysis of the statistical moments of the photoelectron ionization tracks.

Further author information: E-mail: alessia.tortosa@iaps.inaf.it

Space Telescopes and Instrumentation 2018: Ultraviolet to Gamma Ray, edited by Jan-Willem A. den Herder, Shouleh Nikzad, Kazuhiro Nakazawa, Proc. of SPIE Vol. 10699, 106995A · C 2018 SPIE

CCC code: $0277-786 \mathrm{X} / 18 / \$ 18 \cdot$ doi: $10.1117 / 12.2313741$ 
The tracks ionize the gas and an electric field allows electron/ion pairs to drift respectively to the Gas Electron Multiplier (GEM) and top plane. The differential voltage on the GEM induces electron multiplication and the pixelized anode allows a detailed imaging of the track. Imaging can be a useful tool to study the properties of gas diffusion and charge recombination along the drift if the image of the strip produced by an inclined X-ray beam is studied. This detector will be on board the IXPE (Imaging X-ray Polarimetry Explorer) ${ }^{34}$ mission selected by NASA in the framework of the Explorer program scheduled for the launch in 2021.

The use of photoelectric polarimeters is particularly effective in the soft X-ray range, between about 2 and $10 \mathrm{keV}^{567}$. Many astronomical objects, such as active galactic nuclei, pulsars and black holes emit polarized radiation at X-ray wavelengths, in the range in which the GPD is much sensitive. Measuring this polarization will provide fundamental information about the geometry and internal structure of these sources.

We report on tests carried out at INAF-IAPS with a laboratory prototype of the GPD to verify for the first time its capability to measure the polarization of a source with a continuum energy spectrum, that simulates the effect of an astrophysical source.

\section{EXPERIMENTAL SET-UP}
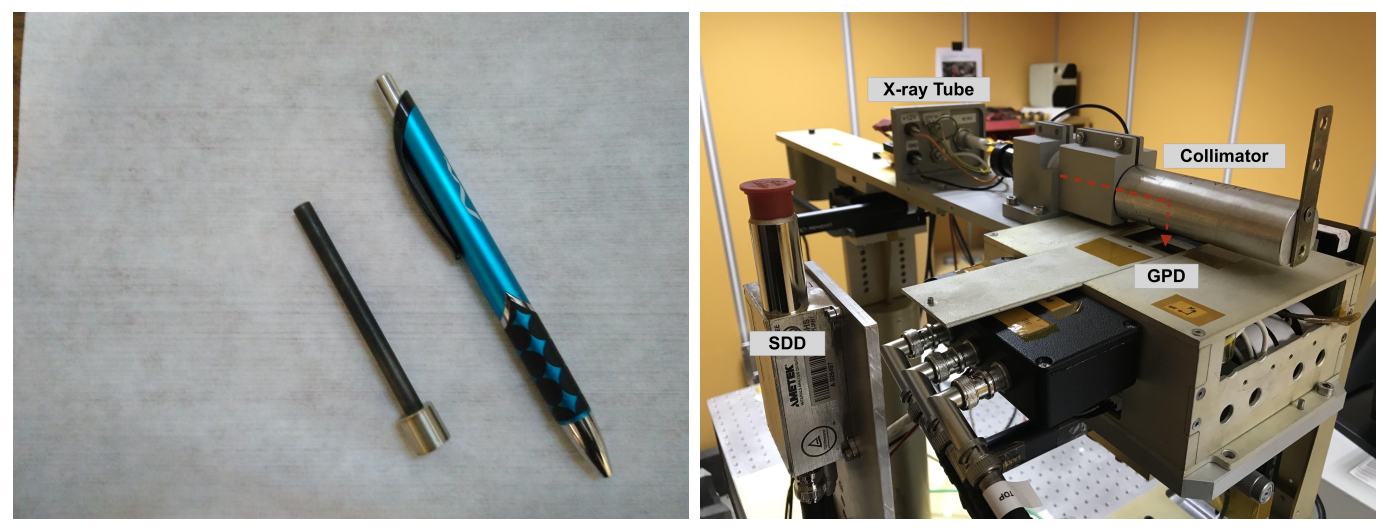

Figure 2. Left panel: lithium scatterer enclosed in Be envelope 1mm thick. Right panel: the experimental set-up.

The laboratory radiation source, with a continuum energy spectrum, is based on Thomson scattering. Xray photons are emitted by an head-on X-ray tube with Tungsten (W) anode, and scattered on a Lithium bar encapsulated in a Beryllium envelope 500 micron thick (see left panel of Fig.2).

When the X-ray photons cross the scatterer, they are scattered among different directions according to Bragg diffraction. The scatterer is hold in a cylindrical collimator made of aluminum. A hole on the lateral surface allow to select X-rays scattered at nearly $90^{\circ}$ towards the detector. The polarization of such scattered photons is nearly $100 \%$. In fact the hole below the collimator is $1 \mathrm{~mm}$ wide and the surface of the collimator is $1 \mathrm{~cm}$ thick. This means that the hole underlies an acceptance cone with a maximum angle $\theta=6^{\circ}$. The polarization is related to this acceptance angle by the following equation:

$$
P=\frac{\sin ^{2}(\theta)}{1+\cos ^{2}(\theta)}
$$

As it is possible to see in Fig3, the photon coming on the GPD from an inclination angle $90^{\circ} \pm \theta / 2$ is $99.5 \%$ polarized. The experimental set-up is shown in right panel of Fig.2. The X-ray tube High Voltage (HV) is set to $10 \mathrm{kV}$. 


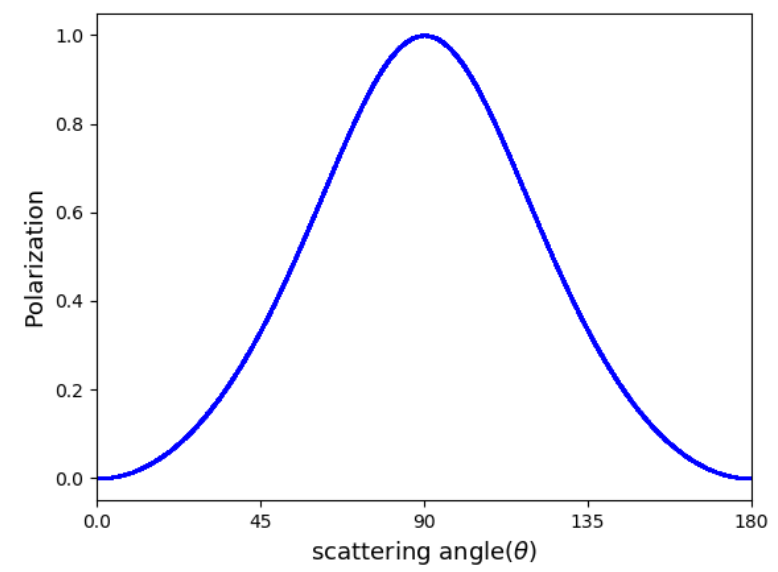

Figure 3. The distribution of the polarization with respect to the acceptance angle following the relation in equation. 1.

\section{RESULTS}

The energy spectrum of the scattering source, measured by means of a Silicon Drift Detector (SDD) (see right panel of Fig.2), is shown in the top panel of Fig.4. Since the spectral resolution of the GPD is about $20 \%$ at $6 \mathrm{keV}$, we measured first the spectrum of the scattering source with the SDD detector (which has a spectral resolution of $\sim 125 \mathrm{eV}$ at $6 \mathrm{keV}$ ) to distinguish all the spectral component of the analysed source, where it is possible to see the fluorescence $\mathrm{K} \alpha$ and $\mathrm{K} \beta$ lines at 3.7 and $4.0 \mathrm{keV}$ of calcium, a contaminant element that is evenly distributed throughout the scatterer. We show, in the bottom panel of Fig.4, the model of an astrophysical energy spectrum (Crab Nebula), convolved with the effective area of the 3 mirrors of IXPE, to highlight the difference in the spectral shape between the continuum X-ray source present in our laboratory and a real astrophysical source. The first one peaks at about $6.5 \mathrm{keV}$, whereas the second one is a power law with spectral index 2 .

The energy spectrum of the scattering source as measured by the GPD is shown in the top panel of Fig.5. The bottom panel of Fig. 5 shows the energy spectrum of the Crab Nebula convoluted with:

- the effective area of the 3 mirrors of IXPE,

- the transparency of the $50 \mu \mathrm{m}$ thick Be entrance window of the GPD,

- the efficiency of the absorption in gas (GPD energy resolution is not taken into account).

After the convolution with the instrument response, the scattering source spectrum still peaks at $6.5 \mathrm{keV}$ while the Crab Nebula spectrum peaks around $2 \mathrm{keV}$ (see Fig.5).

In the energy spectrum of the GPD (see Fig. 5, top panel) the tail below $4 \mathrm{keV}$ and the peak at $2 \mathrm{keV}$ are mainly artifacts due to the detector geometry. Photoelectron tracks truncated by the Beryllium window, or by the Gas Electron Multiplier, GEM (the lower one), or exiting from the sides the active volume (above the readout ASIC), are characterized by an incomplete charge collection.

This effect is less relevant for astrophysical sources, which have typically energy spectra with a larger emission at low energies. However, these events are easily recognized in the charge density plot (see left panel of Fig. 6), where on the x-axis there is the total charge of a track (Pulse Invariant, PI) and on the y-axis there is the number of pixels comprising a track (track size). This events can be removed from the analysis by properly 

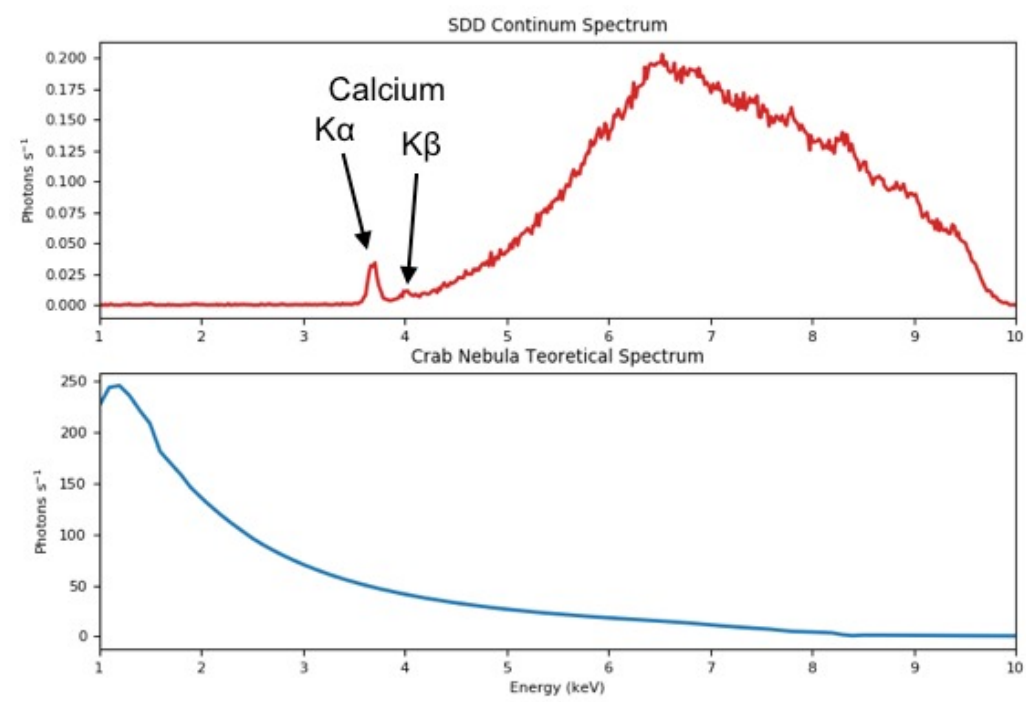

Figure 4. Top panel: scattering source energy spectrum as measured by an SDD. The X-ray tube HV is set to $10 \mathrm{kV}$. Bottom panel: theoretical energy spectrum of the Crab nebula convoluted with the mirrors effective area.
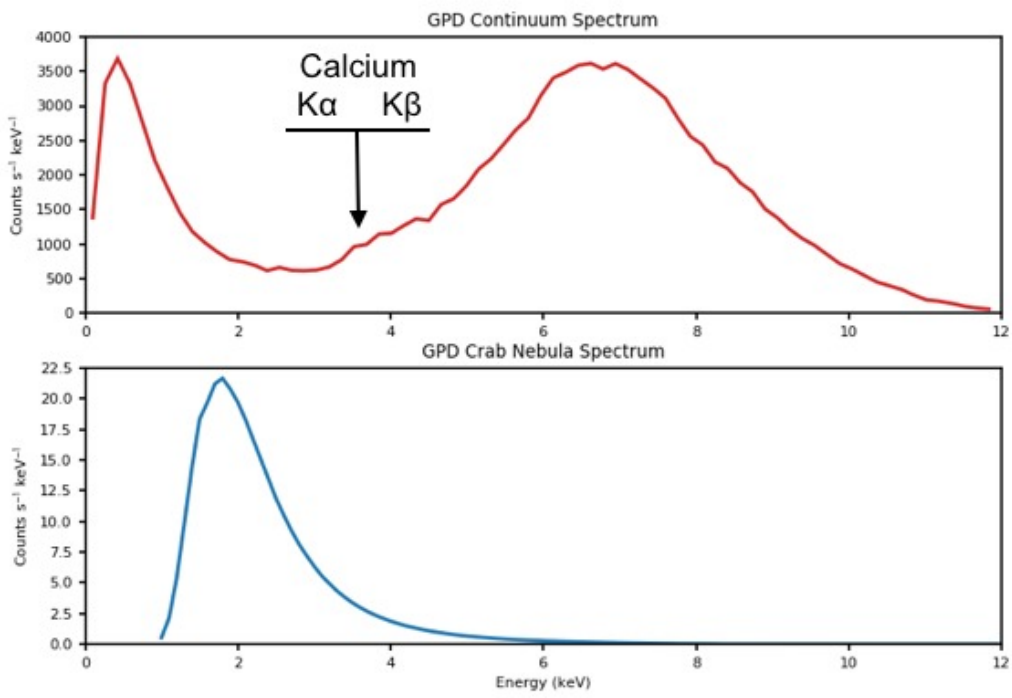

Figure 5. Scattering (top panel) and astrophysical (bottom panel) energy spectra after the convolution with the instrument response.

selecting only those events with the energy of the calcium lines.

The continuum energy spectrum of the scattering source (see Fig. 5, top panel) is divided into 4 energy bins. The modulation factor at the mean energy of events in each bean is calculated. Moreover, tracks with the impact point (photoelectric conversion point) in a frame with a thickness equals to the photoelectron range measured from the image edges are removed from the analysis. Remaining tracks are also filtered depending on ellipticity. 

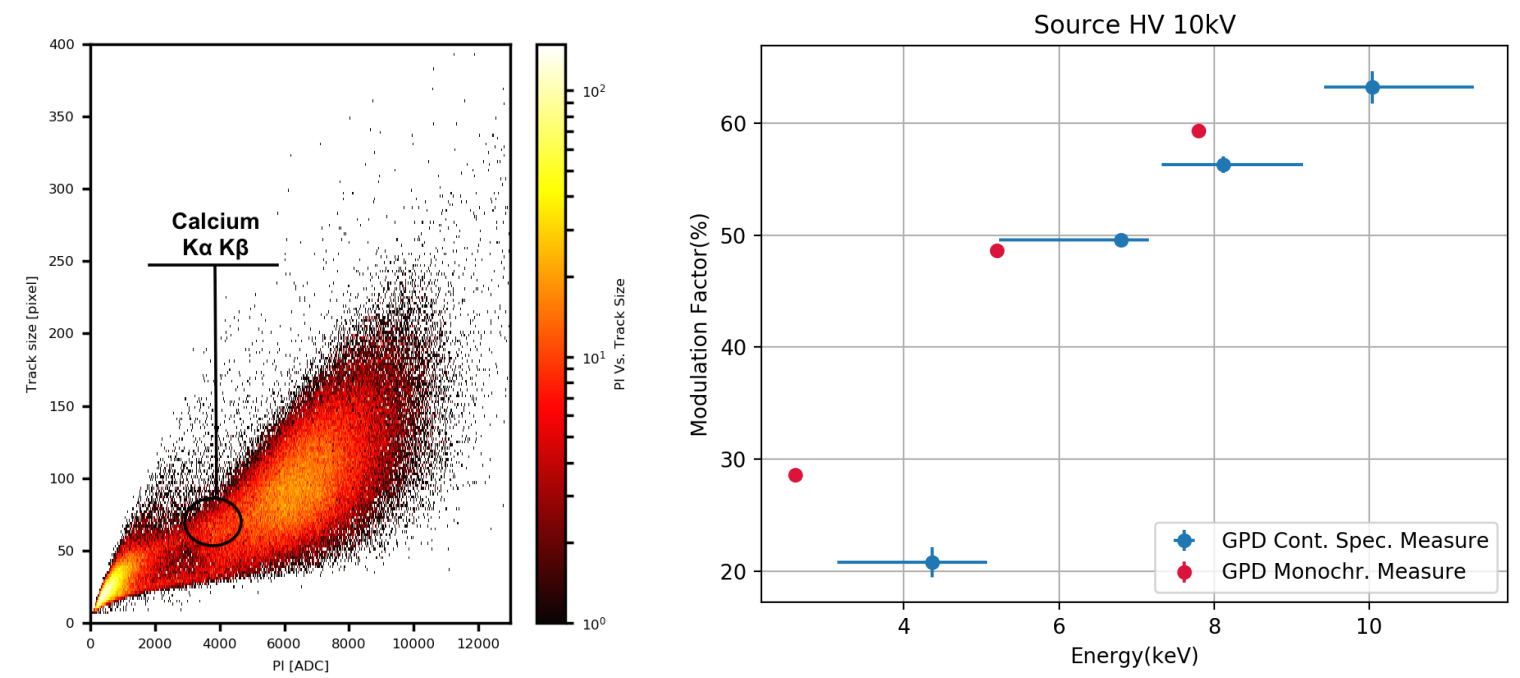

Figure 6. Left panel: the two tails in the region below 4000 PI and 70 pixels correspond mainly to photoelectron tracks truncated by the Beryllium window (the upper one) and by the Gas Electron Multiplier, GEM (the lower one), plus tracks exiting from the sides of the active volume. Right panel: Modulation factor of the scattering source (blue points) and $100 \%$ polarized monochromatic radiation (red points) as a function of energy. In the $3-5$ keV energy bin there are the calcium lines. In the $9.0-11.0 \mathrm{keV}$ range the two points coincide.

This filtering process removes about $20 \%$ of the events. The modulation factor of the scattering source is shown in right panel of Fig. 6 (blue points) and it is compared with the one of $100 \%$ polarized monochromatic radiation (red points) as a function of the energy.

The $3 \mathrm{keV}-5 \mathrm{keV}$ energy bin of the continuum spectrum shows a lower modulation with respect to the monochromatic one due to the presence of the unpolarized fluorescence lines of calcium.

\section{CONCLUSIONS}

The measurement of the linear polarization is one of the hot topics of High Energy Astrophysics. Sensitive $\mathrm{X}$-ray polarimetry promises to solve many different issues in X-ray Astronomy: to disentangle the physics from the geometry by removing degeneracies in models of compact objects in binary systems, magnetars, Supernova Remnants and Pulsar Wind Nebulae. Polarimetry constrains emission mechanisms in blazars and solves the mystery of X-ray emission from cold molecular clouds in the galactic center. Moreover, it can answer to questions of fundamental physics.

We measured for the first time the response of the Gas Pixel Detector to a continuum polarized energy spectrum. This is a very important step for the characterization of this detector that will be on board the IXPE (Imaging X-ray Polarimetry Explorer) mission since the astrophysical sources that IXPE will investigate have a continuum spectrum and not a monochromatic one.

We show the modulation factor of the scattering source compared with $100 \%$ polarized monochromatic radiation as a function of energy. In the 3-5 keV energy bin there are the calcium lines on the scatterer which are less polarized and reduced the modulation factor.

Photoelectron tracks readout by the GPD can be truncated by the edges of the detector active volume and give rise to incomplete charge collection that reduces the modulation of the signal. A proper selection in the analysis 
is needed to avoid this effect. However this effect is less relevant for astrophysical sources, since their energy spectra have a larger emission at lower energy and lower emission at higher energy.

\section{ACKNOWLEDGMENTS}

This activity is funded by the agreement ASI-INAF n. 2017-12-H.0 for the partecipation to the IXPE (Imaging X-ray Polarimeter Explorer) mission approved by NASA. Italian contribution to IXPE mission is supported by the Italian Space Agency through agreement ASI-INAF n.2017-12-H.0 and ASI-INFN agreement n.2017-13-H.0.

\section{REFERENCES}

[1] Weisskopf, M. C., Ramsey, B., O’Dell, S., Tennant, A., Elsner, R., Soffitta, P., Bellazzini, R., Costa, E., Kolodziejczak, J., Kaspi, V., Muleri, F., Marshall, H., Matt, G., and Romani, R., "The Imaging X-ray Polarimetry Explorer (IXPE)," in [Space Telescopes and Instrumentation 2016: Ultraviolet to Gamma Ray], Proc. SPIE 9905, 990517 (July 2016).

[2] Soffitta, P., "IXPE the Imaging X-ray Polarimetry Explorer," in [Society of Photo-Optical Instrumentation Engineers (SPIE) Conference Series], Society of Photo-Optical Instrumentation Engineers (SPIE) Conference Series 10397, 103970I (Aug. 2017).

[3] Costa, E., Soffitta, P., Bellazzini, R., Brez, A., Lumb, N., and Spandre, G., "An efficient photoelectric X-ray polarimeter for the study of black holes and neutron stars," Nature 411, 662-665 (June 2001).

[4] Bellazzini, R., Spandre, G., Minuti, M., Baldini, L., Brez, A., Latronico, L., Omodei, N., Razzano, M., Massai, M. M., Pesce-Rollins, M., Sgró, C., Costa, E., Soffitta, P., Sipila, H., and Lempinen, E., "A sealed Gas Pixel Detector for X-ray astronomy," Nuclear Instruments and Methods in Physics Research A 579, 853-858 (Sept. 2007).

[5] Black, J. K., Baker, R. G., Deines-Jones, P., Hill, J. E., and Jahoda, K., "X-ray polarimetry with a micropattern TPC," Nuclear Instruments and Methods in Physics Research A 581, 755-760 (Nov. 2007).

[6] Muleri, F., Soffitta, P., Baldini, L., Bellazzini, R., Bregeon, J., Brez, A., Costa, E., Frutti, M., Latronico, L., Minuti, M., Negri, M. B., Omodei, N., Pesce-Rollins, M., Pinchera, M., Razzano, M., Rubini, A., Sgró, C., and Spandre, G., "Low energy polarization sensitivity of the Gas Pixel Detector," Nuclear Instruments and Methods in Physics Research A 584, 149-159 (Jan. 2008).

[7] Muleri, F., Soffitta, P., Baldini, L., Bellazzini, R., Brez, A., Costa, E., Fabiani, S., Krummenacher, F., Latronico, L., Lazzarotto, F., Minuti, M., Pinchera, M., Rubini, A., Sgró, C., and Spandre, G., "Spectral and polarimetric characterization of the Gas Pixel Detector filled with dimethyl ether," Nuclear Instruments and Methods in Physics Research A 620, 285-293 (Aug. 2010). 